\title{
An Overview of Computational Studies on Colloidal Semiconductor Nanocrystals
}

\author{
Roberta Pascazio ${ }^{\text {ab }}$, Juliette Zito ${ }^{\text {ab }}$, and Ivan Infante ${ }^{\star a}$
}

\begin{abstract}
In the last two decades, colloidal semiconductor nanocrystals have emerged as a phenomenal research topic due to their size-dependent optoelectronic properties and to their outstanding versatility in many technological applications. In this review, we provide an historical account of the most relevant computational works that have been carried out to understand atomistically the electronic structure of these materials, including the main requirements needed for the preparation of nanocrystal models that align well with the experiments. We further discuss how the advancement of these computational tools has affected the analysis of these nanomaterials over the years. We focus our review on the three main families of colloidal semiconductor nanocrystals: group II-VI and IV-VI metal chalcogenides, group III-V metal pnictogenides and metal halides, in particular lead-based halide perovskites. We discuss the most recent research frontiers and outline the future outlooks expected in this field from a computational perspective.
\end{abstract}

Keywords: Classical Molecular Dynamics · Colloidal semiconductor NCs · Chalcogenides · Density Functional Theory · Perovskites $\cdot$ Pnictogenides
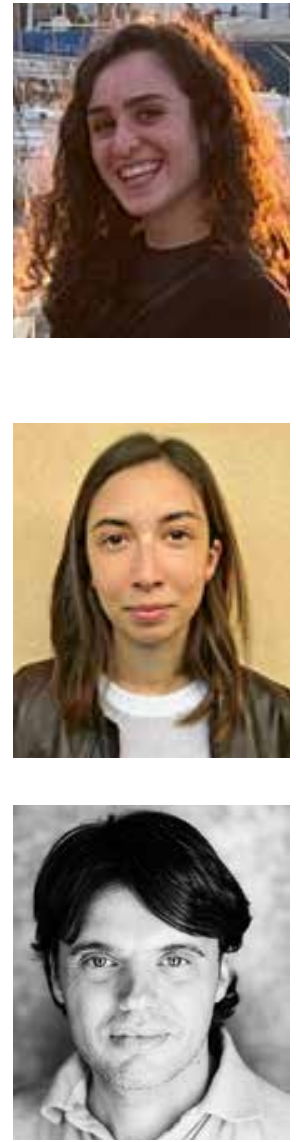

Roberta Pascazio received her Master's Degree in Chemistry from Alma Mater Studiorum - Università di Bologna in Italy in 2020. She is currently a $\mathrm{PhD}$ candidate at the Italian Institute of Technology under the supervision of Ivan Infante and Liberato Manna. Her research addresses the investigation and atomistic description of colloidal semiconductor nanocrystals through computational methodologies.

Juliette Zito received her MS in Chemistry from Università di Genova in Italy in 2019, where she is a $\mathrm{PhD}$ candidate at the Italian Institute of Technology under the supervision of Ivan Infante and Liberato Manna. Her present research focuses on the development and application of computational tools for the atomistic description of semiconductor colloidal nanocrystals.

Ivan Infante received his BSc in Chemistry from Università della Basilicata in Italy in 2001 and his PhD in Theoretical Chemistry from Vrije Universiteit (VU) Amsterdam in 2006. He then carried out postdoctoral work in Geneva, Switzerland, and later started his independent research career at the Universidad del Pais Vasco in Spain thanks to a Juan de la Cierva Grant. In 2014 he was awarded a Vidi Grant from the Netherlands Organization for Scientific Research to establish his own research group as an Assistant Professor at VU Amsterdam. In 2018 he moved to the Istituto Italiano di Tecnologia (IIT), Genova, and leads his own research group. His current research is in the field of colloidal semiconductor nanocrystals, with a special emphasis on characterizing nanocrystal surfaces by combining theoretical calculations and experiments through his many collaborations.

\section{Introduction}

Research on colloidal semiconductor nanocrystals (NCs) has gained growing importance over the last few decades as a consequence of their remarkable characteristics. Nowadays, the many technological applications of NCs span optoelectronics ${ }^{[1]}$ - for instance, they are regarded as key components in the fabrication of light-emitting diodes (LEDs), ${ }^{[2-4]}$ optical sensing, ${ }^{[5,6]}$ instrumentation in the medical/biotechnological field ${ }^{[7,8]}$ (such as lasers ${ }^{[9]}$ and photodetectors $\left.{ }^{[10,11]}\right)$, up to photovoltaics and photocatalysis. ${ }^{[12-22]}$ These nanocrystallites are in fact characterized by several promising features: size-tunable bandgaps, sharp emission spectra, high extinction coefficients, large carrier mobilities ${ }^{[23,24]}$ and in some cases also the possibility to increase the number of excitons emitted through carrier multiplication. ${ }^{[25]}$ Furthermore, these nanocrystals can be regarded as photostable, especially in comparison to organic dyes, because their photoactive part, i.e. the crystalline core, is made of an inorganic semiconductive material. As illustrated in Fig. 1, the inorganic core is capped by organic ligands that mainly ensure NC colloidal stability in apolar solvents. Additionally, these bound ligands help in preventing the formation of trap states, i.e. intragap states emerging from structural defects at the surface, that are deleterious for their light emission efficiency and that in NCs are straightforward to find due to the high surface/volume ratio. ${ }^{[26,27]}$

While the experimental protocols aimed at the synthesis of these compounds are now regarded as commonly established, theoretical calculations still present some challenges, such as the construction of $\mathrm{NC}$ model systems that represent closely those found by experiment and the compromise in the size of the modelled systems, which have to be large enough to avoid strong quantum confinement effects. Increasing the size however puts a 


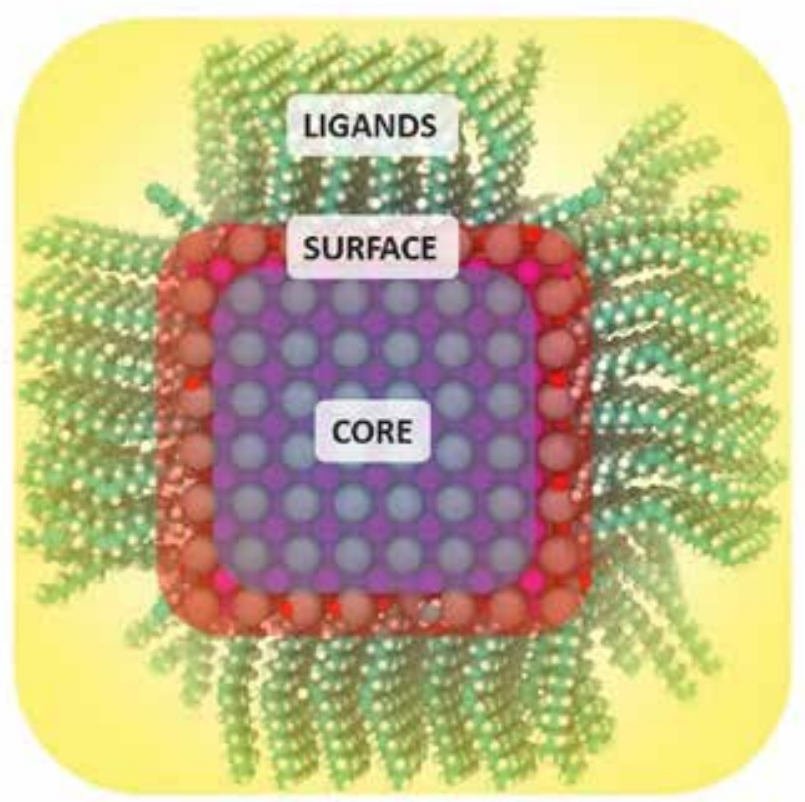

Fig. 1. Balls representation of a perovskite nanocrystal capped by oleate ligands (Cs: grey; Pb: orange; Br: magenta; O: red, C: cyan, $\mathrm{H}$ : white) evidencing three main parts: the inorganic core in light blue, the interfacial surface in red and the shell of molecular ligands in yellow.

strain on the computational requirements, especially for accurate methodologies such as Density Functional Theory (DFT), which scales cubically with the number of atomic basis set functions, i.e. in practice with the number of atoms present in the system. In this review we will therefore discuss the most important achievements obtained in this field from first-principle calculations and an outlook of future developments.

\section{Historical Background}

In Fig. 2, we report a timeline of the most important milestones that have been attained in the colloidal nanocrystals field, from both experimental and computational standpoints. The first reported synthetic endeavors regarding these nanostructures date back to the 1980s. ${ }^{[28-30]}$ Two scientists in particular, Louis Brus $^{[29]}$ and Alexei Ekimov, ${ }^{[30]}$ independently managed to obtain colloidal dispersions of semiconductor crystallites featuring dimensions in the order of a few nanometers, and proved the existence of a correlation between their optical properties and sizes. Successively, in 1993, Murray et al..$^{[31]}$ managed to gain further control on the size distribution and obtained highly monodisperse cadmium chalcogenides nanocrystallites. As a consequence of the structural and electronic relevance of these nanomaterials, over the years, the above synthetic methodologies were soon followed by several theoretical works aimed at the description of their properties. Brus, for instance, made use of tight-binding models ${ }^{[32]}$ to explain the electronic structure and recombination mechanisms of $\mathrm{NCs}$, along with several other features of these crystallites. ${ }^{[32-34]}$ Ekimov, on the other hand, joined forces with his colleague Alexander Efros to develop a model to link the size dependence of the NCs with their optoelectronic features. ${ }^{[35]}$ Some years later, in 2000, Efros published a review ${ }^{[36]}$ detailing a theoretical description of the excitonic and band gap structures in spherical semiconductor nanocrystals. His work gave important details regarding their absorption spectra in line with the experiments, and was soon followed by further investigations on the multiband envelope functions for semiconductor heterostructures. ${ }^{[37,38]}$ From the late 1990s onwards, more accurate atomistic models for the electronic structures of several bulk semiconductors were developed by Delerue and coworkers ${ }^{[39,40]}$ making use of tight binding models with $a d$ hoc parameterizations.

In the following years, a wide range of approaches and levels of theory, ${ }^{[24,41-53]}$ most notably density functional theory, have been applied for the evaluation of the electronic properties of bulk

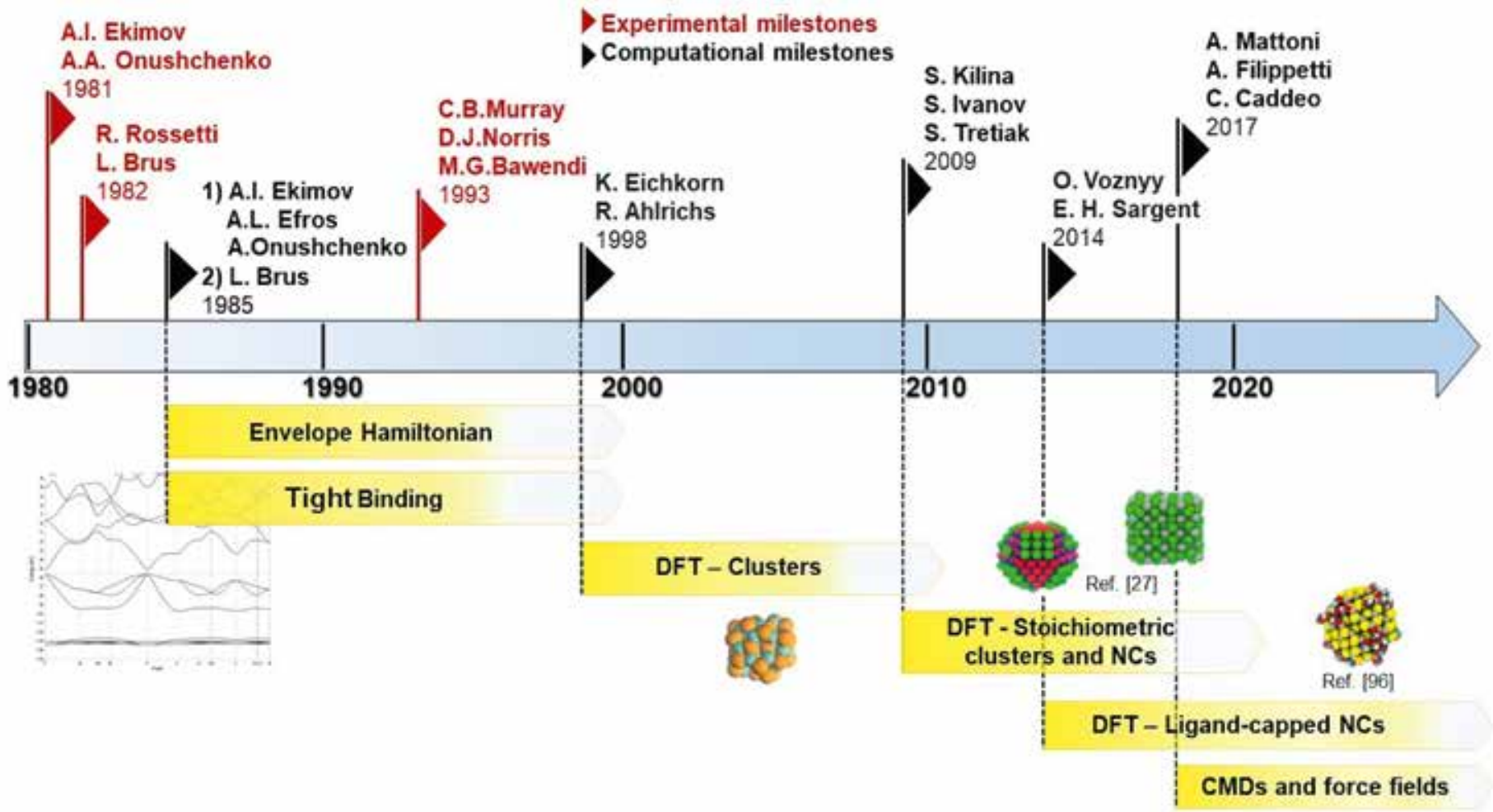

Fig. 2. Timeline of the main experimental and theoretical milestones in the semiconductor nanocrystals field. 
semiconductors, regarded as a litmus test for the characterization of their corresponding finite nanocrystallites. Although these approaches gave valuable insights, they suffered an inherent lack of surface and size-dependence; key features in the description of colloidal semiconductor NCs. As a matter of fact, the first attempts to include such effects involved DFT calculations on small clusters of semiconductors (dimensions of the order of a few unit cells). Unfortunately, until 2010s, DFT could only be effectively employed for the evaluation of strongly confined systems with very limited size (about $1 \mathrm{~nm}$ ) and, in case of molecular dynamic simulations, for fairly brief timespans (usually no longer than the order of 1-5 picoseconds) due to the high computational cost. In the last decade, however, breakthroughs in the modelling of $\mathrm{NCs}$ and the concomitant increase in computational power have paved the way to the atomistic description of NCs counting up to thousands of atoms, thus with sizes and shapes aligned with the experiments.

\section{Preparation of a NC Model for DFT Calculations: The Requirements}

Before diving into the main computational results obtained in this field through the years, we will make a short digression to describe the main requirements to build a $\mathrm{NC}$ model as this is a relevant aspect for those in the $\mathrm{NC}$ community that are interested in starting calculations with colloidal NCs. It is indeed now well established that three main ingredients are necessary for the preparation of $\mathrm{NC}$ models:

- the stoichiometry of the system,

- its overall charge,

- how ligands interact with the surface.

Several experimental[23,46,54,55] investigations have been used over the years to gain insight into the structural features of the experimentally synthesized nanostructures. For instance, Transmission Electron Microscopy (TEM) analysis ${ }^{[56]}$ provides a depiction of NC shapes, while High-Resolution TEM (HRTEM) images ${ }^{[56]}$ of the NCs allows a more thorough visualization of their faceting. The stoichiometric ratios between the elements constituting the nanocrystals, on the other hand, can be determined by means of X-ray photoelectron spectroscopy (XPS), [57] induced coupled plasma (ICP) spectroscopy and other techniques. The atomic ratios of these nanostructures vary depending on the inorganic ions that are left exposed in their outermost layer. For example, in $\mathrm{CsPbBr}_{3} \mathrm{NCs}$, an upper bound for the $\mathrm{Br} / \mathrm{Pb}$ ratio usually corresponds to a $\mathrm{CsBr}$ type of termination, whereas in contrast the lower $\mathrm{Br} / \mathrm{Pb}$ bound is obtain when $\mathrm{abBr}$ layer terminates the NC. A realistic NC model is then realized by tuning the ratios to match those found experimentally. ${ }^{[57]}$

Additionally, in many cases the bottom-up synthetic protocols do not involve harsh conditions in apolar organic solvents, ${ }^{\text {[27] }}$ thus charge neutrality and charge balance conditions are expected. Charge balance in nanocrystal models is easily calculated by means of the charge-orbital balance model, provided by Voznyy et $a l .{ }^{[58]}$ in 2012. In this model, each atom in the core and ligand on the surface of the nanocrystal is assumed to be at its preferential oxidation state defined as the contribution $\mathrm{q}_{\mathrm{i}}$, where $\mathrm{i}$ stands for the type of the atom or ligand under analysis (so, for example, $\mathrm{Pb}$, $\mathrm{Cd}$, Sn will be assumed to have $\mathrm{q}_{\mathrm{i}}=+2$, halogens and carboxylates will have $\mathrm{q}_{\mathrm{i}}=-1$, neutral ligands - such as amines - will be characterized by $\mathrm{q}_{\mathrm{i}}=0$, and so on). The total charge on the nanocrystal $\left(\mathrm{N}_{\text {dop }}\right)$ will then be:

$$
N_{\text {dop }}=-\sum_{i} N_{i} q_{i}
$$

where the $N_{\mathrm{i}}$ in Eqn. (1) accounts for the number of i-type atoms or ligands. This definition of $N_{d o p}$ results in two possible classifications: intrinsic nanocrystals, which occur if the condition $N_{d o p}=0$ is satisfied, and doped or charge unbalanced NCs, corresponding to the $N_{d o p} \neq 0$ case. More specifically, cases with $N_{d o p}>0$ can be ascribed as p-doping, where the electrons have been removed from the valence band (VB) of the material, lowering their energy levels in comparison to the vacuum values. On the other hand, ndoping and consequent increasing of the relative energy levels is obtained if the model features $N_{d o p}<0$.

Finally, the interactions between the core and the ligands can be evaluated within the framework provided by Green's covalent bond classification (CBC) scheme. ${ }^{[59]}$ In this model, the molecular ligands can be defined depending on the number of electrons they can donate to the surface of the core. More specifically, according to the ligand binding motifs introduced for colloidal NCs by Anderson et al., ${ }^{[54]}$ Lewis bases donating two electrons - such as amines and thiols - are defined as L-type ligands. One-electron donors (e.g. phosphonates, carbonates) are defined as X-type ligands, while Lewis acids, usually the $\mathrm{MX}_{\mathrm{n}}$ moiety formed between metal atoms and X-type ligands, are known as Z-type ligands and do not donate any electrons to the surface. Globally, the X-type ligands will saturate the unpaired metal atoms on the surface, each one characterized by valence $v$. As a consequence, as detailed in a work by Giansante et al., ${ }^{[27]}$ a nonstoichiometric $\mathrm{M}_{\mathrm{m}} \mathrm{E}_{\mathrm{n}}$ core saturated with generic ligands can be described by either one of the following notations: by means of the nonstoichiometric molecular formula of the core, accounting for $\mathrm{X}$ and L-type ligands - i.e. $\left[\mathrm{Cs}_{m} \mathrm{Se}_{n}\right] \mathrm{X}_{(\mathrm{m}-\mathrm{n}) \mathrm{v}} \mathrm{L}_{\mathrm{p}}-$ or by means of the formula $[\mathrm{CdSe}]_{\mathrm{n}}\left(\mathrm{CdX}_{\mathrm{v}}\right)_{\mathrm{m}-\mathrm{n}} \mathrm{L}_{\mathrm{p}}$, thus conveniently attaching $\mathrm{Z}$ and L-type ligands to a stoichiometric core.

Once all the above features are properly accounted for, a nanocrystal model can then be straightforwardly built from the corresponding bulk structure of the semiconductor by means of a few simple steps as highlighted in Fig. 3:

- The bulk structure is cut along specific directions to obtain a nanocrystal model of the required dimension that matches the experimental shape and faceting (revealed by TEM and HRTEM images respectively) - see Fig. 3a;

- The surface is terminated by comparing the atomic ratios with the available experimental data (elemental analysis) - see Fig. $3 b$.

- The NC model is charge-balanced according to Eqn. (1) and the charge excess, usually found by a simple cut of the bulk, is eliminated by removing ions from the surface. A brief depiction of the step is provided in Fig. $3 b$.

Finally, when it is desired, organic ligands can be easily added to the surface of the NC model (Fig. 3c). To preserve the chargebalance of the system, the added ligands that carry a given charge contribution should replace surface atoms of corresponding oxidation state (for example carboxylates substituting surface halides, since both feature $q_{i}=-1$ ).

\section{Computational Studies of Colloidal NCs over the Years}

Over the course of this brief review we will summarize the main computational achievements for three families of colloidal semiconductor nanocrystals, namely metal chalcogenides, usually compounds between group VIa anions ( $\mathrm{S}, \mathrm{Se}, \mathrm{Te})$ and group II or IV cations such as $\mathrm{Cd}, \mathrm{Zn}, \mathrm{Pb}$; metal pnictogenides, formed between group III and group V atoms - and metal halides, focusing especially on lead halide perovskites.

\subsection{Group II-VI and IV-VI Metal Chalcogenides}

Both II-VI and IV-VI metal chalcogenides were amongst the first semiconductor nanocrystals to be computationally investigated using DFT methodologies. For instance, in 1998, Eichkorn et al. ${ }^{[60]}$ undertook the first investigation on CdSe clusters proving the validity of DFT in the description of their structural and 


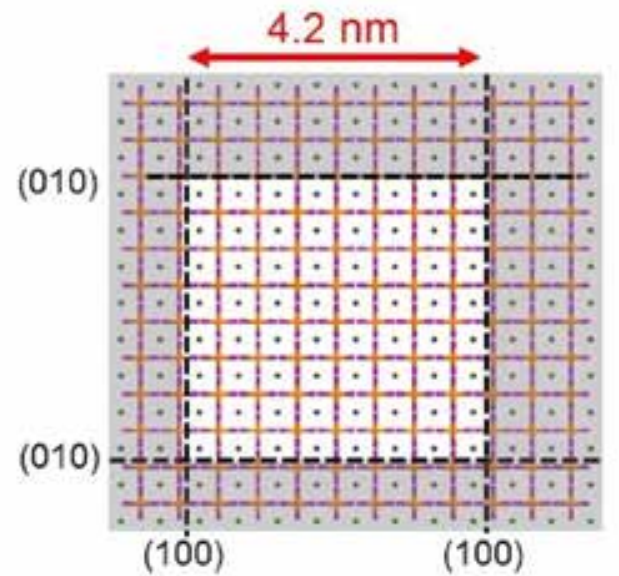

(a)

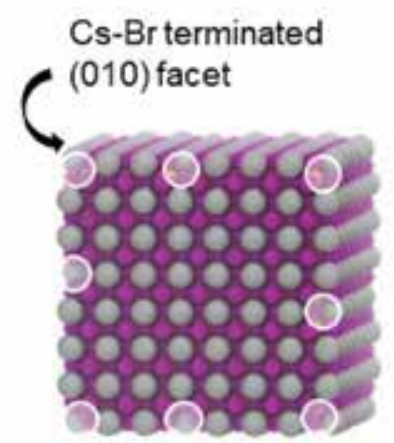

(b)

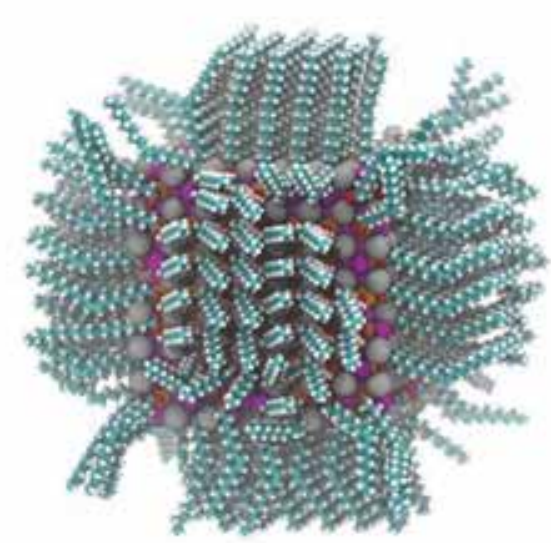

(c)

Fig. 3. Schematic preparation of the NC model. (a) A $4.2 \mathrm{~nm}$ cubic $\mathrm{CsPbBr}_{3}$ core was obtained from the bulk structure by cutting it along the (100) facets, leaving a layer containing $\mathrm{Cs}$ and $\mathrm{Br}$ on the surface; (b) The inorganic core, featuring a stoichiometry of $\mathrm{Cs}_{512} \mathrm{~Pb}_{343} 3 \mathrm{Br}_{1176}, \mathrm{present}$ an excess

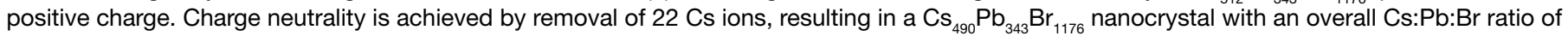
1.4:1:3.4. (c) $80 \%$ of the $\mathrm{Br}$ ions on the surface are replaced with an equal number of oleate anions (OA), as both species feature $\mathrm{q}_{\mathrm{i}}=-1$, resulting in a ligand-capped $\mathrm{Cs}_{490} \mathrm{~Pb}_{343} \mathrm{Br}_{941}(\mathrm{OA})_{235}$ nanocrystal.

electronic features. Successive endeavors by Troparevsky et al. ${ }^{[61]}$ and Deglmann and coworkers ${ }^{[62]}$ attempted to use stoichiometric $\mathrm{Cd}_{\mathrm{x}} \mathrm{Se}_{\mathrm{y}}$ and $\mathrm{Zn}_{\mathrm{x}} \mathrm{Se}_{\mathrm{y}}$ fragments - up to $\mathrm{x}, \mathrm{y}=7$ - as reference models for the same investigational purpose. The analyzed clusters, however, were limited in dimension and were also charge unbalanced, thus failing to provide an accurate description of the electronic structure. Larger, charge-balanced, although still stoichiometric, $\mathrm{Cd}_{\mathrm{x}} \mathrm{Se}_{\mathrm{x}}$ models (up to 90 atoms) were then effectively developed by Pudzer el al.[63] in 2004 to predict the effect of structural relaxation on the electronic properties of the clusters. Follow-up works on the subject ${ }^{[47,48,64]}$ then started comparing and coupling DFT-calculated properties with experimentally acquired data for similarly built models: for instance, the analysis of the main excitation and recombination mechanisms other than a reconstruction of their superficial features and band structures was performed in various studies for both $\mathrm{Pb}_{\mathrm{x}} \mathrm{Se}_{\mathrm{x}}{ }^{[47]}$ and core-shell NC models, ${ }^{[64]}$ as well as for a vast range of zinc compounds. ${ }^{[48]}$ For all these cases, it is worthy to note the effort of applying advanced theoretical methodologies, for example the case of non-adiabatic time-domain molecular dynamic simulations carried out in the picosecond timescale.

In 2009, an article by Kilina et al. ${ }^{[65]}$ employed a DFT scheme on a stoichiometric $\mathrm{Cd}_{33} \mathrm{Se}_{33}$ cluster passivated by small primary amines and phosphines, highlighting the role of L-type ligands in slowing down the non-radiative recombination mechanisms in the cluster. Three years later, in 2012, an article by the same authors ${ }^{[66]}$ outlined the involvement of surface ligands in the acceleration of the electron-phonon mediated exciton relaxation processes displayed by the nanocrystals. Soon after, the development of atomistic simulations emphasized the fundamental role of the inclusion of ligands in the structures in the description of their features. ${ }^{[67]}$ As a consequence, several studies gradually turned their focus towards II-VI and IV-VI NCs passivated by small molecules such as amines, phosphines, and thiols, ${ }^{[68-70]}$ and also towards the investigation of the behavior of more complex ligands - long, aliphatic acetates $^{[23]}$ as well as aromatic amines ${ }^{[71]}$ - on ultrasmall cores, highlighting the strong interaction of these ligands with the inorganic clusters. However, in contrast to the results found for their aliphatic counterparts, such as methylamine, pyridine and aniline were both experimentally and computationally found to introduce ligand-localized states close to the band gap of the $\mathrm{NC}$ - and thus acting as traps - in the electronic structure. The success of these efforts and their good agreement to the experimentally available results soon led to the development of DFT simulations on bigger, more realistic structures: in 2014, for instance, Voznyy et al. ${ }^{[72]}$ were able to provide the first reliable model for the description of a charge balanced, non-stoichiometric $3.6 \mathrm{~nm}$ colloidal CdSe nanocrystal, with size and shape matching CdSe NC synthesized in the lab. This work proposed switching between dynamically activated shallow and deep traps as an atomistic mechanism to justify their photoluminescence quantum yield, and suggested that the inhibition of the blinking process could be obtained through the infiltration of ions in the vacancies. In the same year, Sandeep and co-workers ${ }^{[73]}$ outlined that Z-type ligand removal in $\mathrm{PbSe} \mathrm{NCs}$ via various amines, including chelating ones, affects the necking of NCs in films, ultimately altering their conductivity. A largerscale version of a colloidal PbS NC model, reaching about $5 \mathrm{~nm}$, was then proposed by Zherebetskyy and coworkers ${ }^{[74]}$ to investigate the involvement of superficial hydroxylate groups and water molecules in the passivation of these nanostructures with oleate ligands, which was then confirmed by experimental measurements, such as TEM and XPS. The effect of different type of ligands (L-, $\mathrm{X}$ - and Z-type) on the formation of traps in colloidal II-VI semiconductor nanocrystals was thoroughly examined by Houtepen $e t$ $a l .{ }^{[75]}$ in 2017 . In this work, it was shown that deep trap states are formed through the removal of surface Z-type ligands, creating stable highly uncoordinated chalcogen atoms at the surface (Fig. 4). This work additionally suggested that post-synthetic treatment with Z-type ligands could be effective in healing these surface traps, an approach that was proven to be successful by Kirkwood et $a l .{ }^{[76]}$ A similar approach was used in 2018 by Geiregat et al. ${ }^{[77]}$ for the evaluation of the influence of surface traps on the lasing characteristics of $\mathrm{HgTe}$ zinc-blende nanocrystals capped with methane thiolate. Further steps towards a synergic combination of computational methodologies and experimental techniques had been taken first by Giansante et al. ${ }^{[78]}$ and then by Debellis and coworkers ${ }^{[79]}$ that built efficient models for nonstoichiometric $\mathrm{PbS}$ nanocrystals. NCs of different sizes and capped with formate ligands to mimic oleate ligands from the synthesis, provided an accurate description of their molecular orbitals and band gaps thus giving insight into their absorption properties.

The assessment of DFT quality in the description of II-VI and IV-VI NCs was demonstrated by several benchmark studies over the years. A preliminary work led by Yang et al. ${ }^{[80]}$ in 2008 at- 
tempted to assess the validity of different semiempirical and DFT computational methods on a $\mathrm{Cd}_{2} \mathrm{Se}_{2}$ cluster. The most accurate reproduction of the energetics of the system was found for the B3LYP/LANL2DZ level of theory. A successive paper by Albert et al. ${ }^{\left[{ }^{[1]}\right.}$ on bigger $\mathrm{CdSe} \mathrm{NCs}$ highlighted the contribution provided by polarized basis sets and counterpoise corrections in a precise description of the geometries. A thorough DFT benchmark study was however performed in 2014 by Azpiroz and coworkers ${ }^{[25]}$ over $(\mathrm{MX})_{6}$ quantum dot models, with $\mathrm{M}=\mathrm{Cd}, \mathrm{Zn}$, and $\mathrm{X}=\mathrm{S}$, $\mathrm{Se}, \mathrm{Te}$. Gradient-corrected exchange-correlation functionals like the PBE were found to provide a good compromise between cost and efficiency in geometry optimizations. Nonetheless, despite the increasing computational cost, the overall best performances were displayed for the hybrid PBE0 functional.

\subsection{III-V Metal Pnictogenides}

III-V compound semiconductors have emerged over the years as a very reliable technological alternative to chalcogenides, most notably as a consequence of their very promising optical and electronic properties:[82] for instance, they are widely employed in high-electron-mobility transistors (HEMTs). ${ }^{82,83]}$ Furthermore, the highly tunable band emission and low toxicity found for InP compounds has made them promising candidates for the replacement of red-emitting phosphors in LEDs. ${ }^{[44]}$ In 2006, a screening by Paier and coworkers ${ }^{[84]}$ assessed the performance of several DFT functionals in the evaluation of the main solid state properties for bulk semiconductors containing gallium - most notably GaAs, GaP and $\beta-G a N$. The hybrid HSE functional, in particular, had been found to represent a good compromise between cost and accuracy and was thus employed shortly after by Wadehra et al. ${ }^{[83]}$ and Nicklas et al. ${ }^{[85]}$ for the calculation of the band gaps, the band offsets and the variation of direct-indirect band gap crossovers in bulk III-V alloys in relation to their atomic composition.

Eventually, in 2016, DFT modelling was successfully used in combination with several experimental methodologies to tune both the lattice parameters and the bandgaps in $\operatorname{In}_{\mathrm{x}} \mathrm{Zn}_{\mathrm{y}} \mathrm{P}$ alloy nanocrystals according to necessity ${ }^{[44]}$ and for the computational characterization of the structures and energies of carboxylateligated InP magic-sized nanoclusters, isolated as reaction intermediates during the synthesis of InP nanocrystals. ${ }^{[86]}$ The most recent advancement in the research over this category of materials, however, can be traced to 2017, when a more generic model of anionic passivation and amine-anion co-passivation of the cation-rich surfaces in colloidal $\mathrm{PbS}, \mathrm{CdSe}$ and InAs nanometer-sized nanocrystals was provided by Ko and coworkers. ${ }^{[87]}$ A visual representation of the ligands which have been used in the work for the capping of the InAs NC is provided in Fig. 5.

\subsection{Metal Halides}

Fully inorganic and hybrid organic/inorganic metal halides, especially lead-based perovskites, with chemical formula $\mathrm{APbX}_{3}$ - where $\mathrm{A}$ is a large monovalent cation, such as $\mathrm{Cs}^{+}$and $\mathrm{CH}_{3} \mathrm{NH}_{3}^{+}$ and $\mathrm{X}$ is a halide anion, usually $\mathrm{Br}$, I or, more rarely, $\mathrm{Cl}-$ are regarded as extremely promising candidates in many applications. The involvement of perovskites in photovoltaic devices was first suggested by Kojima et al. ${ }^{[88]}$ in 2009 as a consequence of their highly favorable properties: high conversion efficiency, large carrier mobilities and optimal band gaps. One of the earliest computational investigations, focusing on the electronic and structural features of the bulk form of organic-inorganic $\mathrm{CH}_{3} \mathrm{NH}_{3} \mathrm{PbX}_{3}$ and mixed halide $\mathrm{CH}_{3} \mathrm{NH}_{3} \mathrm{PbI}_{2} \mathrm{X}$ perovskites (with $\mathrm{X}=\mathrm{I}, \mathrm{Br}$, and $\mathrm{Cl}$ ), was led by Mosconi et al. ${ }^{[89]}$ in 2013, and it allowed for a successful comparison of the results with the experimentally available trends. Similar approaches were thus applied in the following years ${ }^{[49-51,90]}$ for both lead and tin-based compounds, extending these evaluations to include the kinetic and structural properties

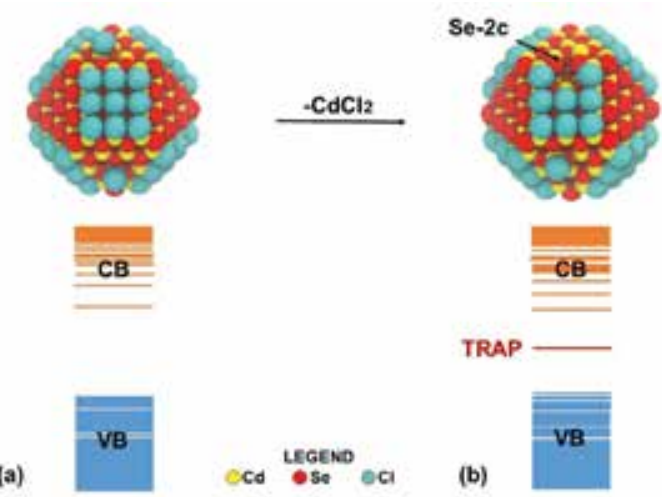

Fig. 4. Depiction of the effect of dicoordinated Se atoms on the valence band (VB) and conduction band (CB) of a CdSe NC. (a) NC without displacements; (b) $\mathrm{NC}$ after removal of a $\mathrm{CdCl}_{2}$ unit (presence of a trap state).

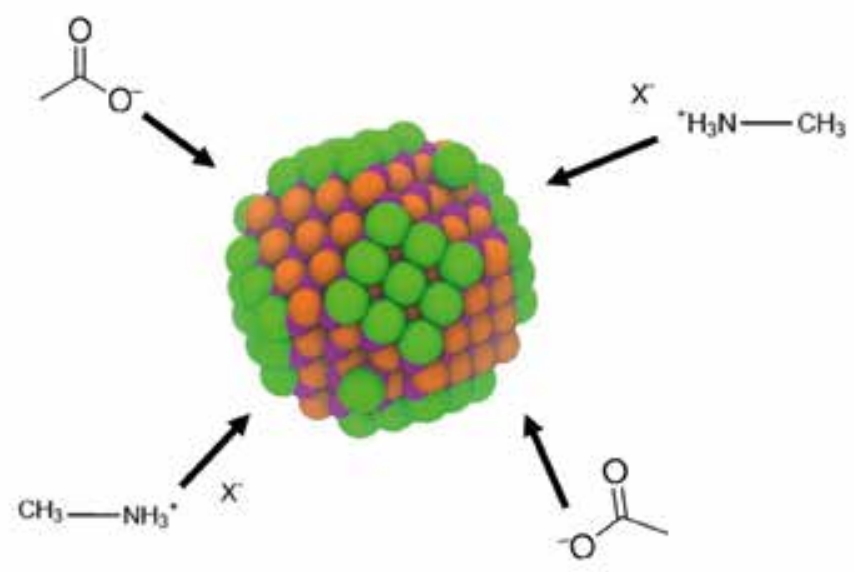

Fig. 5. Depiction of the capping of the InAs NC in ref. [87] by acetate and methylammonium halide co-passivation.

of the first structurally finite cores, ${ }^{[50]}$ such as their ionic migration mechanisms. ${ }^{[53]}$

From 2015 onwards, research over both synthetic strategies for the preparation of colloidal perovskite nanocrystals started to gain increasing relevance. ${ }^{[91]}$ As a matter of fact, several experimental works emphasized their extremely promising properties, such as their high photoluminescence quantum yields (PLQY) and their broadly tunable emission peaks, as well as their exceptional photostability. ${ }^{[74,91-94]}$ Computational simulations focusing on these structures therefore started flourishing in the following years: for instance, in 2016, an article by ten Brinck et al. ${ }^{[95]}$ provided the first atomistic models for the cubic and orthorhombic phases of lead halide perovskite nanocrystals, successfully developing a computational DFT scheme aimed at their atomistic description. The model was effectively validated through the successful calculation of the band gaps and electronic structures of the NCs. A visual representation of the cores which can be obtained from a cubic and orthorhombic halide perovskite lattice is provided in Fig. 6.

Further computational models for perovskite NCs were then developed in 2019[96] to evaluate the influence of the deposition of alkylammonium ligands on the formation of traps states in different, experimentally employed organic solvents. Additional DFT calculations were carried out in the following years, for example proving that alkyl phosphonic ligands on perovskite nanocrystal surfaces ${ }^{[56]}$ stabilize the $\mathrm{PbBr}_{2}$ outer layer rather than the more typical $\mathrm{CsBr}$ layer, commonly found with the oleylammonium/oleate passivation. A more general 
(a)

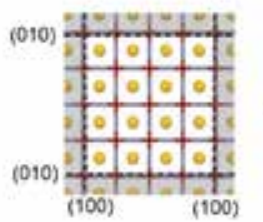

(b)

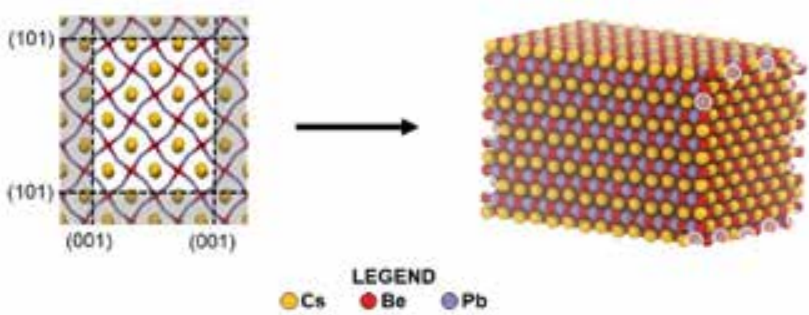

Fig. 6. Charge neutral cores obtained from the cutting of different $\mathrm{CsPbBr}_{3}$ lattices. (a) Cubic lattice; (b) Orthorhombic lattice.

investigation of the role of different solvents on the stabilization of surface ligands was carried out in the following year by Jeon et al., [97] while parallel works by Sercel et al. ${ }^{[98]}$ focused on the analysis of the effect of the shape of the nanocrystals on their electronic and spectroscopic features. Additionally, Bodnarchuk and coworkers ${ }^{[57]}$ examined the main surface degradation mechanisms via displacement of surface ionic pairs and hypothesized feasible restoration pathways for these materials via didodecyldimethyl ammonium bromide (DDAB) post-synthetic treatment, providing good agreement with the experimental observations.

Despite the great promise shown by lead halide perovskite $\mathrm{NCs}$, one of the main drawbacks associated with these materials is their poor colloidal stability, which is strongly limited by the presence of air and moisture and, more importantly, by the intrinsic toxicity of lead. As a consequence, over the last few years, considerable effort has been invested into the search for alternative, non-toxic compounds displaying sufficient stability and matching optical properties. One of the most suitable candidates has been found in double-perovskites (DPs), or elpasolites, a family of materials with general formula $\mathrm{A}_{2} \mathrm{~B}^{+} \mathrm{B}^{3+} \mathrm{X}_{6}$. Several structures of this class - $\mathrm{Cs}_{2} \mathrm{AgBiX}_{6}, \mathrm{Cs}_{2} \mathrm{AgSbX}_{6}(\mathrm{X}=\mathrm{Cl}, \mathrm{Br}$, I) and $\mathrm{Cs}_{2} \mathrm{AgInCl}_{6}$, just to quote a few ${ }^{[93-117]}$ - have been successfully synthesized in the previous years, encouraging a more in-depth computational investigation of their properties. For instance, in 2019, a work by Locardi and coworkers ${ }^{[102]}$ reported the synthesis at the nanoscale of $\mathrm{Bi}$-doped $\mathrm{Cs}_{2} \mathrm{Ag}_{1-x} \mathrm{Na}_{\mathrm{x}} \mathrm{InCl}_{6}$ NCs and coupled a spectroscopic analysis with a DFT-based one to investigate the role of replacing $\mathrm{Na}^{+}$ions with $\mathrm{Ag}^{+}$ones and doping with $\mathrm{Bi}^{3+}$ ions on the PL emission of these materials and proposed a trapped exciton emission mechanism involving localized states on corner-sharing $\mathrm{BiCl}_{6}$ and $\mathrm{AgCl}_{6} \mathrm{Oc}-$ tahedra. Successively, Zhang and coworkers ${ }^{[18]}$ examined the electronic structures of these NCs by leading DFT analysis on $\mathrm{Cs}_{2} \mathrm{Ag}_{0.60} \mathrm{Na}_{0.40} \mathrm{InCl}_{6} \mathrm{NCs}$ models, in the attempt to gain insight on the most effective optimization strategies for the passivation of their surfaces. The progressive displacement of $\mathrm{CsCl}$ pairs from the NCs models evidenced the emergence of deep hole trap states arising from undercoordinated surface $\mathrm{Cl}$ ions even at high surface passivation, thus suggesting the growth of a shell of another semiconductor material over the inorganic core as a feasible strategy to enhance optical performances. A parallel scheme was employed by Zhu et al. ${ }^{[119]}$ on $\mathrm{Cs}_{2} \mathrm{Na}_{1-\mathrm{x}} \mathrm{Ag}_{\mathrm{x}} \mathrm{BiCl}_{6}$ alloy NCs, combining a computational and a spectroscopic analysis to investigate the compositional tuning (in relation to their $\mathrm{Ag}^{+}$content) of the PL emission yield of these materials. Here, deep electron traps were found to emerge from unpassivated $\mathrm{Bi}$ ions at the surface, especially for $\mathrm{Na}^{+}$rich compositions while, upon increasing the $\mathrm{Ag}^{+}$content in the composition of the $\mathrm{NCs}$, these traps became more shallow. More recently, a model for the first epitaxial heterostructure between a lead halide perovskite $\mathrm{NC}$ and a lead chalcohalide one was also proposed. ${ }^{[57,120]}$

\section{Outlook}

In this work, we have depicted a concise timeline of the main computational advances over the years to calculate the electronic and structural properties of colloidal semiconductor nanocrystals. The methodologies described are mostly based on DFT. However, a strong limitation of this approach is the high computational cost, which prevent on the one hand the inclusion of solvent and ligand molecules with the same size and surface coverage as in the experiments and, on the other, performing long timescale simulations using molecular dynamics. For these reasons, it is paramount to move to computationally cheaper alternatives such as those based on classical force fields. Here, an important drawback is the development of accurate force-field parameters that are capable of describing consistently NC models.

Several attempts have however already been made towards this direction: to quote a few examples, in 2017, Mattoni et al. ${ }^{[121]}$ successfully developed a force field aimed at the description of solid-state hybrid metal halide perovskites (MHP) using a combination of Buckingham, Lennard-Jones and Coulombic parameters; more recently, in 2020, Balestra et al..$^{[122]}$ employed a genetic algorithm to model the parameters of a classical force field for the depiction of the structural features and the dynamical properties in cubic and orthorhombic unit cells of all-inorganic metal halide perovskites, showing excellent agreement with experimental findings.

A similar approach has been successfully employed for CdSe nanocrystals by Cosseddu and coworkers ${ }^{[123]}$ in 2017: a stochastic algorithm, the Adaptive Rate Monte Carlo (ARMC), was used to obtain accurate force field parameters for the description of corecore and core-ligand interactions in $\mathrm{CdSe} \mathrm{NCs}$, allowing for the explicit inclusion of the long alkylic chains in the ligands, as well as solvent molecules, in classical molecular dynamics simulations of ligand passivated NCs.

Further calculations on colloidal NCs are therefore expected to make use of the new computational aids provided by classical molecular dynamics studies to extend the investigation both spatially, through simulations involving more realistic systems including solvent and ligand molecules, and temporally, through the evaluation of rarer processes, occurring for more prolonged time scales using accelerated sampling methodologies.

Received: March 17, 2021

[1] T. Nann, W. M. Skinner, ACS Nano 2011, 5, 5291, https://doi.org/10.1021/nn2022974.

[2] H. Cho, S. H. Jeong, M. H. Park, Y. H. Kim, C. Wolf, C. L. Lee, J. H. Heo, A. Sadhanala, N. S. Myoung, S. Yoo, S. H. Im, R. H. Friend, T. W. Lee, Science 2015, 350, 1222, https://doi.org//10.1126/science.aad1818.

[3] S. Wei, Y. Yang, X. Kang, L. Wang, L. Huang, D. Pan, Chem. Commun. 2016, 52, 7265, https://doi.org/10.1039/c6cc01500j.

[4] F. Zhang, H. Zhong, C. Chen, X. G. Wu, X. Hu, H. Huang, J. Han, B. Zou, Y. Dong, ACS Nano 2015, 9, 4533, https://doi.org/10.1021/acsnano.5b01154.

[5] A. Z. Abbasi, F. Amin, T. Niebling, S. Friede, M. Ochs, S. Carregal-Romero, J. M. Montenegro, P. R. Gil, W. Heimbrodt, W. J. Parak, ACS Nano 2011, 5, 21, https://doi.org/10.1021/nn1034026.

[6] D. Jimenez De Aberasturi, J. M. Montenegro, I. Ruiz De Larramendi, T. Rojo, T. A. Klar, R. Alvarez-Puebla, L. M. Liz-Marzán, W. J. Parak, Chem. Mater. 2012, 24, 738, https://doi.org/10.1021/cm202380r.

[7] N. Hildebrandt, ACS Nano 2011, 5, 5286, https://doi.org/10.1021/nn2023123.

[8] I. L. Medintz, H. T. Uyeda, E. R. Goldman, H. Mattoussi, Nat. Mat. 2005, 4, 435, https://doi.org/10.1038/nmat1390. 
[9] S. Yakunin, L. Protesescu, F. Krieg, M. I. Bodnarchuk, G. Nedelcu, M. Humer, G. De Luca, M. Fiebig, W. Heiss, M. V Kovalenko, Nat. Commun. 2015, 6, 8056, https://doi.org/10.1038/ncomms9056.

[10] H. Wei, Y. Fang, P. Mulligan, W. Chuirazzi, H. H. Fang, C. Wang, B. R. Ecker, Y. Gao, M. A. Loi, L. Cao, J. Huang, Nat. Photonics 2016, 10, 333, https://doi.org/10.1038/nphoton.2016.41.

[11] P. Ramasamy, D. H. Lim, B. Kim, S. H. Lee, M. S. Lee, J. S. Lee, Chem. Commun. 2016, 52, 2067, https://doi.org/10.1039/c5cc08643d.

[12] F. Hetsch, X. Xu, H. Wang, S. V Kershaw, A. L. Rogach, J. Phys. Chem. Lett. 2011, 2, 52, https://doi.org/10.1021/jz200802j.

[13] C. Harris, P. V Kamat, ACS Nano 2010, 4, 7321, https://doi.org/10.1021/nn102564x.

[14] C. Harris, P. V Kamat, ACS Nano 2009, 3, 682, https://doi.org/10.1021/nn800848y.

[15] P. V Kamat, Acc. Chem. Res. 1906, 45, 1906 https://doi.org/10.1021/ar200315d.

[16] P. V Kamat, J. Phys. Chem. C 2007, 111, 2834, https://doi.org/10.1021/jp066952u.

[17] P. V Kamat, J. Phys. Chem. C 2008, 112, 18737, https://doi.org/10.1021/jp806791s

[18] O. Voznyy, D. Zhitomirsky, P. Stadler, Z. Ning, S. Hoogland, E. H. Sargent, ACS Nano 2012, 6, 8448, https://doi.org/10.1021/nn303364d.

[19] E. H. Sargent, Nat. Photonics 2012, 6, 133, https://doi.org/10.1038/nphoton.2012.33.

[20] T. P. A. Ruberu, N. C. Nelson, I. I. Slowing, J. Vela, J. Phys. Chem. Lett. 2012, 3, 2798, https://doi.org/10.1021/jz301309d.

[21] C. Wang, R. L. Thompson, J. Baltrus, C. Matranga, J. Phys. Chem. Lett. 2010, 1, 48, https://doi.org/10.1021/jz9000032.

[22] W. S. Yang, J. H. Noh, N. J. Jeon, Y. C. Kim, S. Ryu, J. Seo, S. Il Seok, Science 2015, 348, 1234, https://doi.org/10.1126/science.aaa9272.

[23] H. Choi, J. H. Ko, Y. H. Kim, S. Jeong, J. Am. Chem. Soc. 2013, 135, 5278, https://doi.org/10.1021/ja400948t.

[24] J. H. Lee, Z. Deng, N. C. Bristowe, P. D. Bristowe, A. K. Cheetham, J. Mater. Chem. C 2018, 6, 12252, https://doi.org/10.1039/c8tc04750b.

[25] J. M. Azpiroz, J. M. Ugalde, I. Infante, J. Chem. Theory Comput. 2014, 10, 76, https://doi.org/10.1021/ct400513s.

[26] C. L. Hartley, M. L. Kessler, J. L. Dempsey, J. Am. Chem. Soc. 2021, 143, 1251, https://doi.org/10.1021/jacs.0c10658.

[27] C. Giansante, I. Infante, J. Phys. Chem. Lett. 2017, 8, 5209 , https://doi.org/10.1021/acs.jpclett.7b02193.

[28] A. Henglein, Ber. Bunsengesell. f. Phys. Chem. 1982, 86, 301 https://doi.org/10.1002/bbpc.19820860409.

[29] R. Rossetti, L. Brus, J. Phys. Chem. 1982, 86, 4470, https://doi.org/10.1021/j100220a003.

[30] A. Ekimov, A. Onushchenko, JETP Lett. 1981, 34, 345.

[31] C. B. Murray, D. J. Norris, M. G. Bawendi, J. Am. Chem. Soc. 1993, 115 8706, https://doi.org/10.1021/ja00072a025.

[32] L. E. Brus, J. Phys. Chem. 1986, 90, 2555 https://doi.org/10.1021/j100403a003.

[33] L. E. Brus, J. Chem. Phys. 1984, 80, 4403, https://doi.org/10.1063/1.447218.

[34] L. E. Brus, J. Chem. Phys. 1983, 79, 5566, https://doi.org/10.1063/1.445676.

[35] A. I. Ekimov, A. L. Efros, A. A. Onushchenko, Solid State Commun. 1985 , 56, 921, https://doi.org/10.1016/S0038-1098(85)80025-9.

[36] A. L. Efros, M. Rosen, Annu. Rev. Mater. Sci. 2000, 30, 475 , https://doi.org/10.1146/annurev.matsci.30.1.475.

[37] A. V. Rodina, A. Y. Alekseev, A. L. Efros, M. Rosen, B. K Meyer, Phys. Rev. B - Condens. Matter Mater. Phys. 2002, 65, 1, https://doi.org/10.1103/PhysRevB.65.125302.

[38] A. V. Rodina, L. Efros, M. Rosen, B. K. Meyer, Mater. Sci. Eng. C 2002, 19 , 435, https://doi.org/10.1016/S0928-4931(01)00440-4.

[39] Y. M. Niquet, G. Allan, C. Delerue, M. Lannoo, Appl. Phys. Lett. 2000, 77, 1182, https://doi.org/10.1063/1.1289659.

[40] M. Lannoo, C. Delerue, G. Allan, Phys. Rev. Lett. 1996, 76, 2824 https://doi.org/10.1103/PhysRevLett.74.3415.

[41] A. Puzder, A. J. Williamson, F. Gygi, G. Galli, Phys. Rev. Lett. 2004, 92, 217401, https://doi.org/10.1103/PhysRevLett.92.217401.

[42] M. A. Carignano, A. Kachmar, J. Hutter, J. Phys. Chem. C 2015, 119, 8991, https://doi.org/10.1021/jp510568n.

[43] G. Giorgi, T. Yoshihara, K. Yamashita, Phys. Chem. Chem. Phys. 2016, 18, 27124, https://doi.org/10.1039/c6cp03193e.

[44] F. Pietra, L. De Trizio, A. W. Hoekstra, N. Renaud, M. Prato, F. C. Grozema P. J. Baesjou, R. Koole, L. Manna, A. J. Houtepen, ACS Nano 2016, 10, 4754, https://doi.org/10.1021/acsnano.6b01266.

[45] C. Quarti, E. Mosconi, J. M. Ball, V. D'Innocenzo, C. Tao, S. Pathak, H J. Snaith, A. Petrozza, F. De Angelis, Energy Environ. Sci. 2016, 9, 155, https://doi.org/10.1039/c5ee02925b.

[46] J. Jasieniak, P. Mulvaney, J. Am. Chem. Soc. 2007, 129, 2841 https://doi.org/10.1021/ja066205a.

[47] S. V. Kilina, D. S. Kilin, O. V. Prezhdo, ACS Nano 2009, 3, 93 , https://doi.org/10.1021/nn800674n.
[48] J. M. Azpiroz, I. Infante, X. Lopez, J. M. Ugalde, F. De Angelis, J. Mater. Chem. 2012, 22, 21453, https://doi.org/10.1039/c2jm33744d.

[49] P. Umari, E. Mosconi, F. De Angelis, Sci. Rep. 2014, 4, 1 , https://doi.org/10.1038/srep04467.

[50] Y. He, G. Galli, Chem. Mater. 2014, 26, 5394 https://doi.org/10.1021/cm5026766.

[51] R. Lindblad, D. Bi, B. W. Park, J. Oscarsson, M. Gorgoi, H. Siegbahn, M. Odelius, E. M. J. Johansson, H. Rensmo, J. Phys. Chem. Lett. 2014, 5, 648, https://doi.org/10.1021/jz402749f.

[52] J. M. Azpiroz, E. Mosconi, J. Bisquert, F. De Angelis, Energy Environ. Sci. 2015, 8, 2118, https://doi.org/10.1039/c5ee01265a

[53] C. Eames, J. M. Frost, P. R. F. Barnes, B. C. O’Regan, A. Walsh, M. S Islam, Nat. Commun. 2015, 6, 2, https://doi.org/10.1038/ncomms8497.

[54] N. C. Anderson, M. P. Hendricks, J. J. Choi, J. S. Owen, J. Am. Chem. Soc. 2013, 135, 18536, https://doi.org/10.1021/ja4086758.

[55] R. Grisorio, D. Debellis, G. P. Suranna, G. Gigli, C. Giansante, Angew. Chemie 2016, 128, 6740, https://doi.org/10.1002/ange.201511174.

56] B. Zhang, L. Goldoni, J. Zito, Z. Dang, G. Almeida, F. Zaccaria, J. De Wit, I. Infante, L. De Trizio, L. Manna, Chem. Mater. 2019, 31, 9140 , https://doi.org/10.1021/acs.chemmater.9b03529.

[57] M. I. Bodnarchuk, S. C. Boehme, S. Ten Brinck, C. Bernasconi, Y. Shynkarenko, F. Krieg, R. Widmer, B. Aeschlimann, D Günther, M. V. Kovalenko, I. Infante, ACS Energy Lett. 2019, 4, 63, https://doi.org/10.1021/acsenergylett.8b01669.

[58] O. Voznyy, D. Zhitomirsky, P. Stadler, Z. Ning, S. Hoogland, E. H Sargent, ACS Nano 2012, 6, 8448, https://doi.org/10.1021/nn303364d.

[59] M. L. H. Green, J. Organomet. Chem. 1995, 500, 127, https://doi.org/10.1016/0022-328X(95)00508-N.

[60] K. Eichkorn, R. Ahlrichs, Chem. Phys. Lett. 1998, 288, 235, https://doi.org/10.1016/S0009-2614(98)00306-6.

[61] M. C. Troparevsky, J. R. Chelikowsky, J. Chem. Phys. 2001, 114, 943 , https://doi.org/10.1063/1.1329126.

[62] P. Deglmann, R. Ahlrichs, K. Tsereteli, J. Chem. Phys. 2002, 116, 1585 https://doi.org/10.1063/1.1427718.

[63] A. Puzder, A. J. Williamson, F. Gygi, G. Galli, Phys. Rev. Lett. 2004, 92 1, https://doi.org/10.1103/PhysRevLett.92.217401

[64] J. Jasieniak, P. Mulvaney, J. Am. Chem. Soc. 2007, 129, 2841, https://doi.org/10.1021/ja066205a.

[65] S. Kilina, S. Ivanov, S. Tretiak, J. Am. Chem. Soc. 2009, 131, 7717 https://doi.org/10.1021/ja9005749.

[66] S. Kilina, K. A. Velizhanin, S. Ivanov, O. V. Prezhdo, S. Tretiak, ACS Nano 2012, 6, 6515, https://doi.org/10.1021/nn302371q.

[67] K. Hyeon-Deuk, O. V. Prezhdo, J. Phys. Condens. Matter 2012, 24, https://doi.org/10.1088/0953-8984/24/36/363201

[68] A. M. Abuelela, T. A. Mohamed, O. V Prezhdo, J. Phys. Chem. C 2012 116, 14674, https://doi.org/10.1021/jp303275v.

[69] A. E. Kuznetsov, D. Balamurugan, S. S. Skourtis, D. N. Beratan, J. Phys Chem. C 2012, 116, 6817, https://doi.org/10.1021/jp2109187.

[70] D. Kim, D. H. Kim, J. H. Lee, J. C. Grossman, Phys. Rev. Lett. 2013, 110 , 1, https://doi.org/10.1103/PhysRevLett.110.196802.

[71] J. M. Azpiroz, J. M. Matxain, I. Infante, X. Lopez, J. M. Ugalde, Phys. Chem. Chem. Phys. 2013, 15, 10996, https://doi.org/10.1039/c3cp51687c.

[72] O. Voznyy, E. H. Sargent, Phys. Rev. Lett. 2014, 112, 157401 https://doi.org/10.1103/PhysRevLett.112.157401

[73] C. S. S. Sandeep, J. M. Azpiroz, W. H. Evers, S. C. Boehme, I. Moreels, S. Kinge, L. D. A. Siebbeles, I. Infante, A. J. Houtepen, ACS Nano 2014, 8, 11499, https://doi.org/10.1021/nn504679k.

[74] D. Zherebetskyy, M. Scheele, Y. Zhang, N. Bronstein, C. Thompson, D. Britt, M. Salmeron, P. Alvisatos, L.-W. Wang, Science 2014, 344, 1380, https://doi.org/10.1126/science.1252727

[75] A. J. Houtepen, Z. Hens, J. S. Owen, I. Infante, Chem. Mater. 2017, 29 , 752, https://doi.org/10.1021/acs.chemmater.6b04648.

[76] N. Kirkwood, J. O. V. Monchen, R. W. Crisp, G. Grimaldi, H. A. C. Bergstein, I. Du Fossé, W. Van Der Stam, I. Infante, A. J. Houtepen, J. Am. Chem. Soc. 2018, 140, 15712, https://doi.org/10.1021/jacs.8b07783.

77] P. Geiregat, A. J. Houtepen, L. K. Sagar, I. Infante, F. Zapata, V. Grigel, G. Allan, C. Delerue, D. Van Thourhout, Z. Hens, Nat. Mater. 2018, 17, 35 , https://doi.org/10.1038/NMAT5000.

[78] C. Giansante, I. Infante, E. Fabiano, R. Grisorio, G. P. Suranna, G. Gigli, J. Am. Chem. Soc. 2015, 137, 1875, https://doi.org/10.1021/ja510739q.

[79] D. Debellis, G. Gigli, S. Ten Brinck, I. Infante, C. Giansante, Nano Lett. 2017, 17, 1248, https://doi.org/10.1021/acs.nanolett.6b05087.

[80] P. Yang, S. Tretiak, A. E. Masunov, S. Ivanov, J. Chem. Phys. 2008, 129, 074709, https://doi.org/10.1063/1.2965532.

[81] 'Electronic Structure of Ligated Cdse Clusters: Dependence on DFT Methodology', V. V Albert, S. A. Ivanov, S. Tretiak, S. V Kilina, 2011 https://doi.org/10.1021/jp202510z.

[82] J. A. Alamo, Nature 2011, 479, 3, https://doi.org/10.1038/nature10677.

[83] A. Wadehra, J. W. Nicklas, J. W. Wilkins, Appl. Phys. Lett. 2010, 97, https://doi.org/10.1063/1.3487776. 
[84] J. Paier, M. Marsman, K. Hummer, G. Kresse, I. C. Gerber, J. G. Angyán, J. Chem. Phys. 2006, 124, https://doi.org/10.1063/1.2187006.

[85] J. W. Nicklas, J. W. Wilkins, Appl. Phys. Lett. 2010, 97, https://doi.org/10.1063/1.3485297.

[86] D. C. Gary, S. E. Flowers, W. Kaminsky, A. Petrone, X. Li, B. M. Cossairt, J. Am. Chem. Soc. 2016, 138, 57, https://doi.org/10.1021/jacs.5b13214.

[87] J. H. Ko, D. Yoo, Y. H. Kim, Chem. Commun. 2017, 53, 388, https://doi. org/10.1039/c6cc07933d.

[88] A. Kojima, K. Teshima, Y. Shirai, T. Miyasaka, J. Am. Chem. Soc. 2009, 131, 6050, https://doi.org/10.1021/ja809598r.

[89] E. Mosconi, A. Amat, M. K. Nazeeruddin, M. Grätzel, F. De Angelis, J. Phys. Chem. C 2013, 117, 13902, https://doi.org/10.1021/jp4048659.

[90] G. Giorgi, J. I. Fujisawa, H. Segawa, K. Yamashita, J. Phys. Chem. C 2014, 118, 12176, https://doi.org/10.1021/jp504479c.

[91] L. Protesescu, S. Yakunin, M. I. Bodnarchuk, F. Krieg, R. Caputo, C. H. Hendon, R. X. Yang, A. Walsh, M. V. Kovalenko, Nano Lett. 2015, 15, 3692, https://doi.org/10.1021/n15048779.

[92] P. Ijaz, M. Imran, M. M. Soares, H. C. N. Tolentino, B. Martín-García, C. Giannini, I. Moreels, L. Manna, R. Krahne, J. Phys. Chem. Lett. 2020, 11 , 2079, https://doi.org/10.1021/acs.jpclett.0c00266.

[93] J. Shamsi, A. S. Urban, M. Imran, L. De Trizio, L. Manna, Chem. Rev. 2019, 119, 3296, https://doi.org/10.1021/acs.chemrev.8b00644.

[94] J. M. Azpiroz, J. M. Matxain, I. Infante, X. Lopez, J. M. Ugalde, Phys. Chem. Chem. Phys. 2013, 15, 10996, https://doi.org/10.1039/c3cp51687c.

[95] S. Ten Brinck, I. Infante, ACS Energy Lett. 2016, 1, 1266, https://doi.org/10.1021/acsenergylett.6b00595.

[96] S. Ten Brinck, F. Zaccaria, I. Infante, ACS Energy Lett. 2019, 4, 2739, https://doi.org/10.1021/acsenergylett.9b01945.

[97] S. Jeon, M. C. Jung, J. Ahn, H. K. Woo, J. Bang, D. Kim, S. Y. Lee, H. Y. Woo, J. Jeon, M. J. Han, T. Paik, S. J. Oh, Nanoscale Horizons 2020, 5 , 960, https://doi.org/10.1039/d0nh00029a.

[98] P. C. Sercel, J. L. Lyons, N. Bernstein, A. L. Efros, J. Chem. Phys. 2019, 151, https://doi.org/10.1063/1.5127528

[99] J. C. Dahl, W. T. Osowiecki, Y. Cai, J. K. Swabeck, Y. Bekenstein, M. Asta, E. M. Chan, A. P. Alivisatos, Chem. Mater. 2019, 31, 3134, https://doi.org/10.1021/acs.chemmater.8b04202.

[100] T. Xuan, R. J. Xie, Chem. Eng. J. 2020, 393, 124757, https://doi.org/10.1016/j.cej.2020.124757.

[101] N. Chen, T. Cai, W. Li, K. Hills-Kimball, H. Yang, M. Que, Y. Nagaoka, Z. Liu, D. Yang, A. Dong, C. Y. Xu, R. Zia, O. Chen, ACS Appl. Mater. Interfaces 2019, 11, 16855, https://doi.org/10.1021/acsami.9b02367.

[102] F. Locardi, E. Sartori, J. Buha, J. Zito, M. Prato, V. Pinchetti, M. L.Zaffalon, M. Ferretti, S. Brovelli, I. Infante, L. De Trizio, L. Manna, ACS Energy Lett. 2019, 4, 1976, https://doi.org/10.1021/acsenergylett.9b01274.

[103] S. E. Creutz, E. N. Crites, M. C. De Siena, D. R. Gamelin, Nano Lett. 2018, 18, 1118, https://doi.org/10.1021/acs.nanolett.7b04659.

[104] J. Zhou, X. Rong, P. Zhang, M. S. Molokeev, P. Wei, Q. Liu, X. Zhang, Z. Xia, Adv. Opt. Mater. 2019, 7, 1, https://doi.org/10.1002/adom.201801435.

[105] J. D. Majher, M. B. Gray, T. A. Strom, P. M. Woodward, Chem. Mater. 2019, 31, 1738, https://doi.org/10.1021/acs.chemmater.8b05280.

[106] B. Yang, X. Mao, F. Hong, W. Meng, Y. Tang, X. Xia, S. Yang, W. Deng, K. Han, J. Am. Chem. Soc. 2018, 140, 17001, https://doi.org/10.1021/jacs.8b07424.
[107] K. Z. Du, W. Meng, X. Wang, Y. Yan, D. B. Mitzi, Angew. Chem. Int. Ed. 2017, 56, 8158, https://doi.org/10.1002/anie.201703970.

[108] A. Karmakar, M. S. Dodd, S. Agnihotri, E. Ravera, V. K. Michaelis, Chem. Mater. 2018, 30, 8280, https://doi.org/10.1021/acs.chemmater.8b03755.

[109] Y. Liu, Y. Jing, J. Zhao, Q. Liu, Z. Xia, Chem. Mater. 2019, 31, 3333, https://doi.org/10.1021/acs.chemmater.9b00410.

[110] S. Vasala, M. Karppinen, Prog. Solid State Chem. 2015, 43, 1, https://doi. org/10.1016/j.progsolidstchem.2014.08.001.

[111] K. Lv, S. Qi, G. Liu, Y. Lou, J. Chen, Y. Zhao, Chem. Commun. 2019, 55, 14741, https://doi.org/10.1039/c9cc07397c.

[112] S. Khalfin, Y. Bekenstein, Nanoscale 2019, 11, 8665 , https://doi.org/10.1039/c9nr01031a.

[113] B. Yang, F. Hong, J. Chen, Y. Tang, L. Yang, Y. Sang, X. Xia, J. Guo, H. He, S. Yang, W. Deng, K. Han, Angew. Chem. Int. Ed. 2019, 58, 2278, https://doi.org/10.1002/anie.201811610.

[114] W. Lee, S. Hong, S. Kim, J. Phys. Chem. C 2019, 123, 2665, https://doi.org/10.1021/acs.jpcc.8b12146.

[115] Y. Bekenstein, J. C. Dahl, J. Huang, W. T. Osowiecki, J. K. Swabeck, E. M. Chan, P. Yang, A. P. Alivisatos, Nano Lett. 2018, 18, 3502, https://doi.org/10.1021/acs.nanolett.8b00560.

[116] Y. Zhang, T. Shah, F. L. Deepak, B. A. Korgel, Chem. Mater. 2019, 31, 7962, https://doi.org/10.1021/acs.chemmater.9b02149.

[117] F. Locardi, M. Cirignano, D. Baranov, Z. Dang, M. Prato, F. Drago, M. Ferretti, V. Pinchetti, M. Fanciulli, S. Brovelli, L. De Trizio, L. Manna, J. Am. Chem. Soc. 2018, 140, 12989, https://doi.org/10.1021/jacs.8b07983.

[118] B. Zhang, M. Wang, M. Ghini, A. E. M. Melcherts, J. Zito, L. Goldoni, I. Infante, M. Guizzardi, F. Scotognella, I. Kriegel, L. De Trizio, L. Manna, ACS Mater. Lett. 2020, 2, 1442, https://doi.org/10.1021/acsmaterialslett.0c00359.

[119] D. Zhu, J. Zito, V. Pinchetti, Z. Dang, A. Olivati, L. Pasquale, A. Tang, M. L. Zaffalon, F. Meinardi, I. Infante, L. De Trizio, L. Manna, S. Brovelli, ACS Energy Lett. 2020, 5, 1840, https://doi.org/10.1021/acsenergylett.0c00914.

[120] M. Imran, L. Peng, A. Pianetti, V. Pinchetti, J. Ramade, J. Zito, F. Di Stasio, J. Buha, S. Toso, J. Song, I. Infante, S. Bals, S. Brovelli, L. Manna, J. Am. Chem. Soc. 2021, 143, 1435, https://doi.org/10.1021/jacs.0c10916.

[121] A. Filippetti, A. Mattoni, Phys. Rev. B - Condens. Matter Mater. Phys. 2014, 89, 1, https://doi.org/10.1103/PhysRevB.89.125203.

[122] S. R. G. Balestra, J. M. Vicent-Luna, S. Calero, S. Tao, J. A. Anta, J. Mater. Chem. A 2020, 8, 11824, https://doi.org/10.1039/d0ta03200j.

[123] S. Cosseddu, I. Infante, J. Chem. Theory Comput. 2017, 13, 297, https://doi.org/10.1021/acs.jctc.6b01089.

\section{License and Terms}

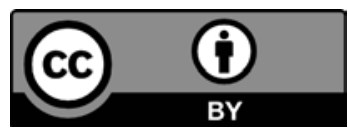

This is an Open Access article under the terms of the Creative Commons Attribution License CC BY 4.0. The material may not be used for commercial purposes.

The license is subject to the CHIMIA terms and conditions: (http:// chimia.ch/component/sppagebuilder/?view = page \&id=12).

The definitive version of this article is the electronic one that can be found at https://doi.org/10.2533/chimia.2021.427 